\title{
Cataract extraction and patient vision- related quality of life: a cohort study
}

U Javed ${ }^{1}, K^{\prime}$ McVeigh ${ }^{2}$, NW Scott ${ }^{3}$ and A Azuara-Blanco ${ }^{4}$

\begin{abstract}
Aims Cataract surgery is one of the most common surgeries performed, but its overuse has been reported. The threshold for cataract surgery has become increasingly lenient; therefore, the selection process and surgical need has been questioned. The aim of this study was to evaluate the changes associated with cataract surgery in patient-reported vision-related quality of life (VR-QoL).
\end{abstract}

Methods A prospective cohort study was conducted. Consecutive patients referred to cataract clinics in an NHS unit in Scotland were identified. Those listed for surgery were invited to complete a validated questionnaire (TyPE) to measure VR-QoL pre- and postoperatively. TyPE has five different domains (near vision, distance vision, daytime driving, night-time driving, and glare) and a global score of vision. The influence of pre-operative visual acuity (VA) levels, vision, and lens status of the fellow eye on changes in VR-QoL were explored.

Results A total of 320 listed patients were approached, of whom 36 were excluded. Among the 284 enrolled patients, 229 (81\%) returned the questionnaire after surgery. Results revealed that the mean overall vision improved, as reported by patients. Improvements were also seen in all subdomains of the questionnaire.

Conclusion The majority of patients appear to have improvement in patient-reported VR-QoL, including those with good preoperative VA and previous surgery to the fellow eye. VA thresholds may not capture the effects of the quality of life on patients. This information can assist clinicians to make more informed decisions when debating over the benefits of listing a patient for cataract extraction.

Eye (2015) 29, 921-925; doi:10.1038/eye.2015.70; published online 15 May 2015

\section{Introduction}

Cataract surgery is one of the most common elective surgical procedures in the $\mathrm{UK}^{1}$ and worldwide. $^{2}$ The complication rate of cataract surgery is small. However, as described by Malik et $a l^{3}$ overuse of cataract surgery has been reported in many studies. ${ }^{4-10}$ The thresholds for cataract surgery have become increasingly lenient ${ }^{11,12}$ with a large number of patients undergoing cataract surgery with good vision. In patients with good vision, the need for surgery and potential benefits are dubious. As stated in the study by Malik et al, ${ }^{3}$ small changes in threshold criteria can have a significant effect on the number of patients indicated for surgery. The need or selection process for cataract surgery has therefore been questioned.

The assessment of cataract surgery outcomes has primarily been by measuring change in visual acuity (VA). ${ }^{3}$ VA before surgery has been reported to be $6 / 12$ or better in $33 \%,{ }^{3}$ twothirds, ${ }^{13}$ and $69 \%{ }^{12}$ of cataract patients. These statistics are important, as vision of $6 / 12$ or better is considered to be within driving standards ${ }^{14}$ and some tax-funded health-care systems restrict cataract surgery to those with VA worse than $6 / 12 .{ }^{15}$ The provision of unnecessary cataract surgery has been suggested by Sletteberg et al, ${ }^{8}$ who reported that one in six patients listed for cataract surgery felt that their vision was not impaired enough for surgery to be needed at that time. With the increasing rates of cataract surgery among the population, the effectiveness and cost-effectiveness of cataract operations need to be evaluated. Patients with minimal visual impairment are less likely to experience the benefits of cataract surgery. Another study reported that after surgery 27\% of patients felt that their vision had either stayed the same or was worse after the procedure. ${ }^{11}$

The aim of this project was to profile patients listed for cataract extraction within a NHS unit
${ }^{1}$ Wishaw Resource Centre, Wishaw, Scotland

${ }^{2}$ Ophthalmology Department, Royal United Hospital, Bath, England

${ }^{3}$ Medical Statistics Team, Division of Applied Health Sciences, University of Aberdeen, Aberdeen, Scotland

${ }^{4}$ Centre for Experimental Medicine, Queen's University Belfast, Belfast, Northern Ireland

Correspondence:

U Javed, Wishaw Resource Centre, 48-54 Roberts

Street, Wishaw ML2 7JF, Scotland

Tel: +1698354031.

E-mail: u.javed@nhs.net

Received: 5 October 2014 Accepted in revised form: 13 March 2015 Published online: 15 May 2015 
and to evaluate the vision-related quality of life (VR-QoL) and visual function at the time of surgery, and to compare them with changes after the operation.

\section{Materials and methods}

A prospective cohort study was designed. Patients suffering with cataracts that were significant enough to consider extraction were identified. Consecutive patients on the assessment list between September 2011 and October 2011 were invited to participate in this study and asked to complete a questionnaire at the time of the surgical pre-assessment clinic and again 4 months postoperatively. Patients were asked to complete the questionnaire upon entering the cataract pre-assessment clinic, before the installation of mydriatic drops. The same questionnaire was posted to patients 4 months postoperatively. The local Ethics Committee approved the study.

Patient demographics, Snellen VA, ocular co-morbidity, other medical co-morbidities, and surgical status of the fellow eye were all documented.

The inclusion criteria included the following: English speaking, age $>50$ years, and referral for cataract surgery by an Optometrist, GP, or Ophthalmologist. The exclusion criteria were as follows: cognitive impairment; significant ocular co-morbidities affecting vision (such as complete blindness or trauma); those undergoing concomitant procedures for other ophthalmologic conditions; and previous cataract surgery on either eye within the past 8 weeks.

Cognitive assessment was measured using the 4-item Abbreviated Mental Test, ${ }^{16}$ which asked the following questions: (1) Age, (2) Date of birth, (3) Place, and (4) Year. Those scoring $<4$ indicated cognitive impairment and were excluded from analysis.

We evaluated the VR-QoL using a validated questionnaire, TyPE. ${ }^{13,17}$ With regard to the scoring information of the TyPE Questionnaire (see Supplementary Appendix), we used what seems to be the most commonly presented approach to scoring the instrument. ${ }^{17,18}$ 'Overall vision' was assessed using Q1 of the TyPE instrument. This was rescaled so that 0 represented poor vision and 100 represented good vision. The 12 items in sections 2 and 3 were used to derive both a total TyPE score and various sub-scales. The number of items per sub-scale was either one (daytime driving, night-time driving), two (distance vision, near vision), or four (glare). The total TyPE score is an unweighted score of the 12 items. It has also been suggested that the item on night-time driving is dropped from the total TyPE score to improve the psychometric properties of the scale; therefore, this 11-item total score has also been presented.
Scale scores for respondents selecting 0 ('Don't do for other reason') for any constituent item or with missing values for any constituent item were not calculated, except when over half the items had been answered, in which case missing values were imputed using the other scale items.

Scores were described before and after the cataract operation and, for those with valid scores at both time points, the mean change was also calculated. Post-surgery scores were also described for four categories of vision referring to the VA of the elected and fellow eye before the operation. For VA 'good' vision was defined as 6/12 or better; 'poor' vision was defined as $6 / 18$ or worse. Using this categorisation, patients were separated into four sub-groups (elective/fellow): Good/Good, Good/ Poor, Poor/Good, and Poor/Poor. Scores were also described according to whether this was the first or second cataract surgery.

\section{Results}

Of 320 listed patients approached, 36 were excluded. The reasons for exclusion are detailed in Table 1.

Of the remaining 284 patients listed for surgery, 229 responded before and after operation (81\%). The average age was 74 years, 139/229 (61\%) were female, 133/229 (58\%) had right-eye surgery, and 172/229 (75\%) had first cataract surgery. The VA in the surgical eye was $6 / 12$ or better in $69 / 229(30 \%)$ patients, between $6 / 18$ and $6 / 36$ in $114 / 229(50 \%)$ patients, and $6 / 60$ or worse in $46 / 229$ (20\%) patients. Five questionnaires were completed inadequately and were excluded from analysis, leaving 224 for analysis of post-operative outcomes.

Mean overall vision at baseline according to the TyPE questionnaire was 26.2 (SD, 16.4) in the 229 included patients (Table 2). Post-operatively, this figure improved to 60.2 (SD, 25.6). In all, 47/224 (21\%) patients suffered with one or more ocular co-morbidities.

Table 3 shows that the best score in post-operative overall vision was for the Poor (elective eye)/Good (fellow eye) group. The Poor/Good vision group reported better outcomes than the Good/Good group in all dimensions.

Table 1 Reasons for exclusion

\begin{tabular}{lc}
\hline Reason for exclusion & Number \\
\hline Age <50 years & 1 \\
Cognitive impairment & 8 \\
Ocular co-morbidity & 8 \\
Incorrect completion of questionnaire & 9 \\
Surgery still awaited & 5 \\
Surgery declined by patient & 1 \\
Deceased & 4 \\
\hline
\end{tabular}


Tables 4 and 5 show the change in vision outcomes associated with patients who had either first or second cataract surgery. This shows that patients noted a similar subjective improvement in overall VA following first and second surgeries with an improvement in overall vision of 33.7 (SD 27.6) after first eye surgery and 34.2 (SD 29.0) after second eye surgery.

Table 2 Mean (SD) (n) scores (pre, post, and change)

\begin{tabular}{lccr}
\hline & Pre & Post & Change \\
\hline Overall vision & $26.2(16.4)[229]$ & $60.2(25.6)[224]$ & $33.8(27.9)[224]$ \\
Distance vision & $62.8(25.1)[228]$ & $79.3(24.2)[219]$ & $16.7(31.0)[218]$ \\
Near vision & $55.0(26.5)[229]$ & $71.8(27.3)[224]$ & $16.8(34.7)[224]$ \\
Daytime driving & $69.9(28.6)[132]$ & $85.8(24.7)[127]$ & $19.7(30.1)[118]$ \\
Night-time driving & $47.7(30.8)[122]$ & $72.7(28.5)[118]$ & $25.0(35.5)[105]$ \\
Glare & $59.1(23.2)[228]$ & $72.0(23.4)[211]$ & $13.5(25.9)[210]$ \\
Total TyPE score (11 items) & $60.4(20.4)[228]$ & $75.4(22.0)[215]$ & $15.4(25.1)[215]$ \\
Total TyPE score (12 items) & $59.9(20.5)[228]$ & $75.0(22.0)[216]$ & $15.6(25.3)[216]$ \\
\hline
\end{tabular}

Table 3 Mean (SD) (n) post scores by category of visual acuity (elected eye/fellow eye)

\begin{tabular}{lcccc}
\hline & Good/good & Good/poor & Poor/good & Poor/poor \\
\hline Overall vision & $57.5(25.3)[63]$ & $56.3(23.9)[4]$ & $63.2(25.0)[121]$ & $54.9(27.9)[36]$ \\
Distance vision & $73.6(26.1)[64]$ & $81.3(23.9)[4]$ & $83.8(21.4)[119]$ & $73.4(27.8)[32]$ \\
Near vision & $69.4(29.6)[65]$ & $75.0(20.4)[4]$ & $74.2(25.9)[121]$ & $67.3(28.5)[34]$ \\
Daytime driving & $84.4(24.5)[40]$ & $25.0[1]$ & $89.9(19.4)[74]$ & $70.8(41.1)[12]$ \\
Night-time driving & $71.8(27.0)[39]$ & $25.0[1]$ & $76.4(25.8)[70]$ & $50.0(44.3)[8]$ \\
Glare & $67.6(24.7)[60]$ & $81.3(13.0)[4]$ & $75.0(21.8)[113]$ & $68.6(25.8)[34]$ \\
Total TyPE score (11 items) & $71.5(23.9)[63]$ & $78.6(23.3)[3]$ & $78.9(19.6)[116]$ & $70.1(24.4)[33]$ \\
Total TyPE score (12 items) & $71.3(23.6)[63]$ & $77.8(24.7)[3]$ & $78.3(19.7)[117]$ & $69.9(24.7)[33]$ \\
\hline
\end{tabular}

Table 4 Mean (SD) (n) scores for first surgery (pre, post, and change)

\begin{tabular}{lccc}
\hline & Pre & Post & Change \\
\hline Overall vision & $24.0(15.9)[171]$ & $57.8(24.8)[167]$ & $33.7(27.6)[161]$ \\
Distance vision & $61.1(25.3)[170]$ & $77.5(24.0)[162]$ & $16.8(31.2)[161]$ \\
Near vision & $54.5(26.6)[171]$ & $70.1(27.5)[167]$ & $15.7(36.0)[167]$ \\
Daytime driving & $65.8(29.7)[98]$ & $84.9(24.4)[91]$ & $22.1(30.5)[86]$ \\
Night-time driving & $45.6(31.7)[90]$ & $70.5(28.2)[83]$ & $24.0(34.7)[76]$ \\
Glare & $58.0(23.3)[170]$ & $70.7(24.0)[160]$ & $12.7(26.7)[159]$ \\
Total TyPE score (11 items) & $59.3(20.6)[170]$ & $73.4(22.1)[161]$ & $14.6(25.7)[161]$ \\
Total TyPE score (12 items) & $58.8(20.7)[170]$ & $73.1(22.0)[161]$ & $14.9(26.0)[161]$ \\
\hline
\end{tabular}

Table 5 Mean (SD) (n) scores for second surgery (pre, post, and change)

\begin{tabular}{lcrr}
\hline & Pre & Post & Change \\
\hline Overall vision & $32.8(16.4)[58]$ & $67.1(26.8)[57]$ & $34.2(29.0)[57]$ \\
Distance vision & $67.7(24.1)[58]$ & $84.4(24.5)[57]$ & $16.4(31.0)[57]$ \\
Near vision & $56.4(26.4)[58]$ & $76.8(26.2)[57]$ & $20.0(30.8)[57]$ \\
Daytime driving & $81.6(21.6)[34]$ & $88.2(25.7)[36]$ & $13.3(28.4)[32]$ \\
Night-time driving & $53.9(27.8)[32]$ & $77.9(28.9)[35]$ & $27.6(38.0)[29]$ \\
Glare & $62.2(22.8)[58]$ & $76.0(21.1)[51]$ & $16.1(23.4)[51]$ \\
Total TyPE score (11 items) & $63.7(19.7)[58]$ & $81.1(20.9)[54]$ & $17.7(23.1)[54]$ \\
Total TyPE score (12 items) & $63.2(19.5)[58]$ & $80.4(21.2)[55]$ & $17.4(23.5)[55]$ \\
\hline
\end{tabular}




\section{Discussion}

This study was conducted to evaluate changes in patientreported VR-QoL and visual function in an NHS hospital.

Several questionnaires have been developed to measure quality-of-life outcomes in patients with cataracts. These include Activities of Daily Vision Scale, Visual Disability Assessment, and VF-14. However, these are limited in their assessment of quality of life associated with visual disability in cataract patients. ${ }^{13}$ The VF-14 also has a limitation in that it is developed for use in the USA and its applicability to the UK populations may be limited. The TyPE Questionnaire was developed as a collaboration between Interstudy, Inc. (Minneapolis, MN, USA) and the Association of University Professors of Ophthalmology. ${ }^{17}$ TyPE was chosen for this study as it showed high testretest reliability and very high Spearman and Kappa coefficients. ${ }^{18}$ It was considered that 4 months after surgery would be the period when the greatest impact of surgery would be apparent and when it would be more likely that visual recovery and rehabilitation would have stabilised. ${ }^{18}$

In our study, following surgery, mean scores for all sub-scales (near vision, distance vision, day-time driving, night-time driving, and glare) improved. This was noted in patients with both poor VA and apparently good preoperative vision. Overall vision improved from 26.2 to 60.2 using Q1 of the TyPE Questionnaire. For all subscales, improvements were noted, although relative changes in scores should be carefully interpreted, as a change in one sub-scale may not be directly comparable to changes in another. It should also be acknowledged that there are large SDs for some TyPE sub-scales, which may indicate substantial variability in reported scores.

With an average age of 74 years, a cognitive assessment was done pre-operatively to exclude patients with possible cognitive impairment. This selection of patients has helped prevent inappropriate completion of questionnaires, and it is a strength of this study.

Questionnaires were completed before a decision was made to proceed with cataract surgery, and the decision was irrespective of the questionnaire scoring. It cannot be excluded that patients may have scored their vision worse than it may have actually been, as they may have thought that scoring well may result in them not having the surgery.

As expected, the Poor (elective)/Good (fellow eye) group [pre] scored better patient-reported outcomes in overall vision and in all sub-groups [post] than patients with Good/Good vision. This may reflect the phenomenon of response shift. Patients undergoing surgery in their second eye reported better baseline results in all sub-scales but experienced similar improvements between first vs second eye cataract surgery.

Cataract extraction is still being offered in the NHS in patients with relatively good vision (such as $6 / 12$ or better). In our study, 30\% $(67 / 224)$ of the participants were in this category. Although patients with poor preoperative vision reported the largest improvement in visual function, we also could detect improvement in those with good pre-operative vision.

The risks and complications associated with cataract surgery should be considered when listing patients for surgery, particularly in cases with good VA.

Endophthalmitis is an uncommon but major cause of post-cataract surgery blindness, with one study reporting an incidence rate of $0.128 \% .{ }^{19}$ Pseudophakic retinal detachment incidence rates after surgery have been reported to be $1.17 \% .{ }^{20}$ Although not common, these complications are relevant because of the potentially poor outcomes and the large number of surgeries done, with cataract removal being the most common elective ophthalmic surgical procedure performed worldwide. $^{2}$

It is recognised that VA alone may not be an ideal measure of the overall visual limitations. Currently, there is no standardised assessment of patient-reported VRQoL to help evaluate the need for cataract surgery. The use of VA threshold criteria for cataract surgery in taxfunded healthcare systems can have a significant effect on the number of patients who are selected for surgery but may not capture well the limitations in patients' quality of life. Other factors that should be considered are the type of intraocular lens used. This was demonstrated in the study by Denoyer et al, ${ }^{20,21}$ in which an aspherical intraocular lens with a negative spherical aberration resulted in reduced ocular spherical aberration and improved mesopic contrast sensitivity, and led to better subjective quality of vision. The lens choice can also be affected by glare disability, as studied by Pandita et al. ${ }^{22}$ This information, alongside the individuals' personal profile, could be used to help clinicians make more informed decisions.

In summary, this study provides evidence on improvement in visual function patient-reported VR-QoL in a large cohort of patients undergoing cataract surgery. This includes those with poor and good vision in the eye elected for surgery, as well as those with good vision and previous surgery in the fellow eye. Best outcomes were noted in patients with poor vision in the elective eye. The results of VA and VR-QoL can be taken into consideration to assist clinicians to make more informed and standardised assessments when debating the benefits or risks of listing a patient for cataract extraction. 


\section{Summary}

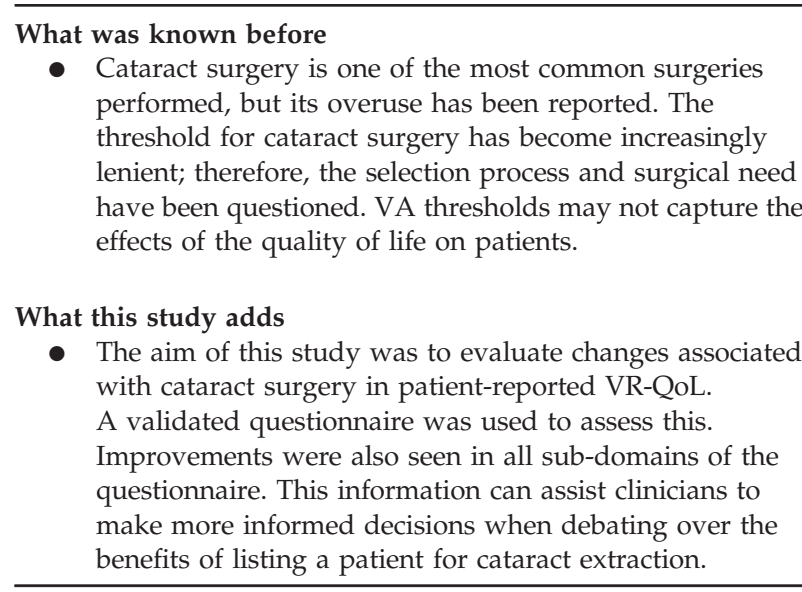

\section{Conflict of interest}

The authors declare no conflict of interest.

\section{Acknowledgements}

We acknowledge the following people, without whose support the study would not have been possible to complete: Dr Jane Harcourt (Clinical Services Manager, Department of Ophthalmology, Aberdeen Royal Infirmary), Paul Chua (Specialist Registrar, Aberdeen Royal Infirmary), and the nursing staff at the cataract clinic.

\section{References}

1 Department of Health and Social Services National Statistics. Census Tables 2001. Hospital Episode Statistics. London, 2001.

2 Nagy Z, Takacs A, Filkorn T, Sarayba M. Initial clinical evaluation of an intraocular femtosecond laser in cataract surgery. J Refract Surg 2009; 25: 1053-1060.

3 Malik R, Frost NA, Sparrow JM, Hale L. The Bristol cataract listing survey: profile of listed patients with visual acuity 6/12 or better. Eye (Lond) 2005; 19(4): 406-410.

4 Alonso J, Espallargues M, Andersen TF, Cassard SD, Dunn E, Bernth-Petersen P et al. International applicability of the VF-14. An index of visual function in patients with cataracts. Ophthalmology 1997; 104(5): 799-807.

5 Damiano AM, Steinberg EP, Cassard SD, Bass EB, Diener-West M, Legro MW et al. Comparison of generic versus disease-specific measures of functional impairment in patients with cataract. Med Care 1995; 33(4 Suppl): AS120-AS130.

6 Mildon D, Noertjojo K, Rollins D, Blicker J, Law F, Courtright $\mathrm{P}$ et al. Cataract surgery at the Vancouver Eye Care Centre: do patient indications meet provincial clinical practice guidelines? Can J Ophthalmol 2003; 38(3): 199-206.
7 O'Day DM. Socioeconomics viewpoint: the need for an update of the clinical practice guideline on cataract [review]. Arch Ophthalmol 1995; 113(6): 718-720.

8 Sletteberg O, Hovding G, Bertelsen T. Do we operate too many cataracts? The referred cataract patients' own appraisal of their need for surgery. Acta Ophthalmol Scand 1995; 73(1): 77-80.

9 Tobacman JK, Lee P, Zimmerman B, Kolder H, Hilborne L, Brook R. Assessment of appropriateness of cataract surgery at ten academic medical centers in 1990. Ophthalmology 1996; 103(2): 207-215.

10 Tobacman JK, Zimmerman B, Lee P, Hilborne L, Kolder H, Brook RH. Visual acuity following cataract surgeries in relation to preoperative appropriateness ratings. Med Decis Making 2003; 23(2): 122-130.

11 Wright CJ, Chambers K, Robens-Paradise Y. Evaluation of indications for outcomes of elective surgery. CMAJ 2002; 167(5): 461-466.

12 Chua P, Mustafa MS, Scott NW, Kumarasamy M, Azuara-Blanco A. Relationship between socioeconomic deprivation or urban/rural residence and visual acuity before cataract surgery in Northern Scotland. Eur J Ophthalmol 2013; 23(6): 831-835.

13 Pesudovs K, Caudle LE, Rees G, Lamoureux E. Validity of a visual impairment questionnaire in measuring cataract surgery outcomes. J Cataract Refract Surg 2008; 34(6): 925-933.

14 Direct Gov. UK 2014 Driving eyesight requirements. Available at https://www.gov.uk/driving-eyesight-rules.

15 Tuulonen A, Salminen H, Linna M, Perkola M. The need and total cost of Finnish eyecare services: a simulation model for 2005-2040. Acta Ophthalmol 2009; 87(8): 820-829.

16 Swain DG, O'Brien AG, Nightingale PG. Cognitive assessment in elderly patients admitted to hospital: the relationship between the shortened version of the abbreviated mental test and the abbreviated mental test and mini-mental state examination. Clin Rehabil 2000; 14(6): 608-610.

17 Javitt JC, Jacobson G, Schiffman RM. Validity and reliability of the Cataract TyPE Spec: an instrument for measuring outcomes of cataract extraction. Am J Ophthalmol 2003; 136(2): 285-290

18 Lundström M, Pesudovs K. Questionnaires for measuring cataract surgery outcomes. J Cataract Refract Surg 2011; 37(5): 945-959.

19 Mehran T, Behrens A, Newcomb RL, Nobe MY, Saedi G, Sweet PM et al. Acute endophthalmitis following cataract surgery. A systematic review of the literature. Arch Ophthalmol 2005; 123(5): 613-620.

20 Russell M, Gaskin B, Russell D, Polkinghome PJ. Pseudophakic retinal detachment after phacoemulsification cataract surgery: Ten-year retrospective review. J Cataract Refract Surg 2006; 32(3): 442-445.

21 Denoyer A, Marie-Laure LL, Majzoub S, Pierre-Jean P. Quality of vision after cataract surgery after Tecnis Z9000 intraocular lens implantation: Effect of contrast sensitivity and wavefront aberration improvements on the quality of daily vision. J Cataract Refract Surg 2007; 33(2): 210-216.

22 Pandita D, Raj SM, Vasavada VA, Vasavada VA, Kazi NS, Vasavada AR. Contrast sensitivity and glare disability after implantation of AcrySof IQ Natural aspherical intraocular lens: Prospective randomised masked clinical trial. J Cataract Refract Surg 2007; 33(4): 603-610. 